\title{
APPLICATION OF FRACTIONAL ORDER THEORY OF THERMOELASTICITY TO A 1D PROBLEM FOR A SPHERICAL SHELL
}

\author{
WALEED E. RASLAN \\ Mansoura University, Department of Mathematics and Engineering Physics, Mansoura, Egypt \\ e-mail:w_raslan@yahoo.com
}

\begin{abstract}
In this work, we apply the fractional order theory of thermoelasticity to a one-dimensional problem of distribution of thermal stresses and temperature in a generalized thermoelastic medium in the form of a spherical shell subjected to sudden change in the temperature of its external boundary. Laplace transform techniques are used to solve the problem. Numerical results are computed and represented graphically for the temperature, displacement and stress distributions.
\end{abstract}

Keywords: fractional calculus, spherical shell, thermoelasticity

\section{Introduction}

Biot (1956) formulated theory of coupled thermoelasticity to eliminate the paradox inherent in the classical uncoupled theory that elastic changes have no effect on temperature. Lord and Shulman (1967) introduced theory of generalized thermoelasticity with one relaxation time by using the Maxwell-Cattaneo law of heat conduction instead of the conventional Fourier law. The heat equation associated with this theory is hyperbolic and hence eliminates the paradox of infinite speeds of propagation inherent in both the uncoupled and coupled theories of thermoelasticity. Sherief and El-Maghraby (2003, 2005) solved some crack problems for this theory. Sherief and Hamza $(1994,1996)$ obtained a solution to axisymmetric problems in spherical and cylindrical regions. Sherief and Ezzat (1994) obtained the solution in form of a series. Sherief et al. (2005) extended this theory to deal with micropolar materials. That theory was extended to deal with viscoelastic effects by Sherief et al. (2011). Lately, Sherief and Hussein (2012) developed theory of generalized poro-thermoelasticity.

Fractional calculus has been successfully used to modify many existing models of physical processes, see Hilfer (2000), Sherief et al. (2012), Tenreiro et al. (2013). One can state that the whole theory of fractional derivatives and integrals was established in the 2nd half of the 19th century. A good review of the subject can be found in Podlubny (1998), Kaczorek (2011), Kaczorek and Rogowski (2015). Caputo and Mainardi (1971a,b) and Caputo (1974) found a good agreement with experimental results by making use of fractional derivatives for description of viscoelastic materials and established the connection between the fractional derivatives and the theory of linear viscoelasticity. Adolfsson et al. (2005) constructed a new fractional order model of viscoelasticity.

Povstenko (2009) made a review of thermoelasticity that uses a fractional heat conduction equation and proposed and investigated new models that incorporate fractional derivatives (Povstenko, 2005, 2011). Recently, the fractional order theory of thermoelasticity was derived by Sherief et al. (2010). It was a generalization of both coupled and generalized theories of thermoelasticity. Some contributions to that theory are the works by Raslan (2015), Sherief and Abd El-Latief (2014a,b, 2015). 
The aim of the present work is to solve a 1D problem for a spherical shell of a homogeneous, isotropic, thermoelastic medium occupying the region $a \leqslant r \leqslant b$ subjected to thermal shock, using the fractional theory of thermoelasticity. The main reason behind the introduction of the fractional theory is that it predicts a retarded response to physical stimuli, as is found in nature and as opposed to the instantaneous response predicted by the generalized theory of thermoelasticity (Raslan, 2015).

\section{Formulation of the problem}

In this work, we consider a 1D problem for a spherical shell of a homogeneous, isotropic, thermoelastic medium occupying the region $a \leqslant r \leqslant b$, using the fractional theory of thermoelasticity. The outer surface of the shell is taken to be traction free and is subjected to thermal shock that is a function of time. The inner surface of the shell is thermally isolated by a rigid material.

From physics of the problem, all functions will depend on the radial distance $r$ and time $t$ only. The displacement vector has only one non-zero component $u(r, t)$ in the radial direction.

The governing equations, in the absence of body forces and heat sources, are given by (Sherief et al., 2010)

$$
\begin{array}{ll}
(\lambda+2 \mu) \frac{\partial e}{\partial r}-\gamma \frac{\partial T}{\partial r}=\rho \frac{\partial^{2} u}{\partial t^{2}} & k \nabla^{2} T=\left(\frac{\partial}{\partial t}+\tau_{0} \frac{\partial^{\alpha+1}}{\partial t^{\alpha+1}}\right)\left(\rho c_{E} T+\gamma T_{0} e\right) \\
\sigma_{r r}=\lambda e+2 \mu \frac{\partial u}{\partial r}-\gamma\left(T-T_{0}\right) & q_{r}+\tau_{0} \frac{\partial^{\alpha} q_{r}}{\partial t^{\alpha}}=-k \frac{\partial T}{\partial r}
\end{array}
$$

where $T$ is the absolute temperature, $\rho$ is density, $\lambda$ and $\mu$ are Lamé's constants and $\gamma=\alpha_{t}(3 \lambda+2 \mu)$, where $\alpha_{t}$ is the coefficient of linear thermal expansion. $T_{0}$ is the reference temperature assumed to be such that $\left|\left(T-T_{0}\right) / T_{0}\right| \ll 1$ and $\alpha, \tau_{0}$ are constants such that $\tau_{0}>0,0 \leqslant \alpha \leqslant 1, c_{E}$ is the specific heat per unit mass in the absence of deformation and $k$ is the thermal conductivity, $\sigma_{r r}$ is the normal stress component, $q_{r}$ is the component of the heat flux vector in the radial direction, and $e$ is the cubical dilatation given by

$$
e=\frac{1}{r^{2}} \frac{\partial}{\partial r}\left(r^{2} u\right)
$$

The operator $\nabla^{2}$ in the above equations is given by

$$
\nabla^{2}=\frac{\partial^{2}}{\partial r^{2}}+\frac{2}{r} \frac{\partial}{\partial r}=\frac{1}{r^{2}} \frac{\partial}{\partial r}\left(r^{2} \frac{\partial}{\partial r}\right)
$$

We shall use the following non-dimensional variables

$$
\begin{aligned}
& r^{*}=c \eta r \quad u^{*}=c \eta u \quad t^{*}=c^{2} \eta t \quad \tau_{0}^{*}=c^{2 \alpha} \eta^{\alpha} \tau_{0} \\
& \theta^{*}=\frac{\gamma\left(T-T_{0}\right)}{\lambda+2 \mu} \quad \sigma_{r r}^{*}=\frac{\sigma_{r r}}{\mu} \quad q^{*}=\frac{\gamma}{k(\lambda+2 \mu)} q
\end{aligned}
$$

where

$$
c=\sqrt{\frac{\lambda+2 \mu}{\rho}} \quad \eta=\frac{\rho c_{E}}{k}
$$

The governing equations, in non-dimensional form, are given by (with the asterisk dropped for convenience)

$$
\begin{array}{ll}
\frac{\partial^{2} u}{\partial t^{2}}=\frac{\partial e}{\partial r}-\frac{\partial \theta}{\partial r} & \nabla^{2} \theta=\left(\frac{\partial}{\partial t}+\tau_{0} \frac{\partial^{\alpha+1}}{\partial t^{\alpha+1}}\right)(\theta+\varepsilon e) \\
\sigma_{r r}=\left(\beta^{2}-2\right) e+2 \frac{\partial u}{\partial r}-\beta^{2} \theta & q_{r}+\tau \frac{\partial^{\alpha} q_{r}}{\partial t^{\alpha}}=-\frac{\partial \theta}{\partial r}
\end{array}
$$


where

$$
\varepsilon=\frac{T_{0} \gamma^{2}}{\lambda+2 \mu} k \eta \quad \beta^{2}=\frac{\lambda+2 \mu}{\mu}
$$

In the above equation, the time fractional derivative of the order $\alpha$ used is taken to be in the sense of the Caputo fractional derivative.

We assume that the boundary conditions have the form

$$
\begin{array}{ll}
u(a, t)=0 & q_{r}(a, t)=0 \\
\sigma_{r r}(b, t)=0 & \theta(b, t)=f(t)
\end{array}
$$

The initial conditions are taken to be homogeneous, i.e. we take

$$
\begin{aligned}
& \left.u(r, t)\right|_{t=0}=\left.\frac{\partial u(r, t)}{\partial t}\right|_{t=0}=\left.0 \quad \theta(r, t)\right|_{t=0}=\left.\frac{\partial \theta(r, t)}{\partial t}\right|_{t=0}=0 \\
& \left.\sigma_{r r}(r, t)\right|_{t=0}=\left.\frac{\partial \sigma_{r r}(r, t)}{\partial t}\right|_{t=0}=0
\end{aligned}
$$

\section{Solution in the Laplace transform domain}

Applying the Laplace transform with the parameter $s$ (denoted by the overbar) defined by the relation

$$
\bar{f}(r, s)=\int_{0}^{\infty} e^{-s t} f(r, t) d t
$$

to both sides of equations (2.3), we get the following equations

$$
\begin{array}{ll}
s^{2} \bar{u}=\frac{\partial \bar{e}}{\partial r}-\frac{\partial \bar{\theta}}{\partial r} & \nabla^{2} \bar{\theta}=\left(s+\tau_{0} s^{\alpha+1}\right)(\bar{\theta}+\varepsilon \bar{e}) \\
\bar{\sigma}_{r r}=\frac{\beta^{2}-2}{r} \bar{u}+\beta^{2} \frac{\partial \bar{u}}{\partial r}-\beta^{2} \bar{\theta} & \bar{q}_{r}=\frac{-1}{1+\tau s^{\alpha}} \frac{\partial \bar{\theta}}{\partial r}
\end{array}
$$

Applying the operator $\frac{1}{r^{2}} \frac{\partial}{\partial r}\left(r^{2} \ldots\right)$ to equation $(3.2)_{1}$, we obtain

$$
\left(\nabla^{2}-s^{2}\right) \bar{e}=\nabla^{2} \bar{\theta}
$$

Eliminating $\bar{\theta}$ between equations $(3.2)_{2}$ and (3.3), we get

$$
\left\{\nabla^{4}-\nabla^{2}\left[s^{2}+(1+\varepsilon)\left(s+\tau_{0} s^{\alpha+1}\right)\right]+s^{3}\left(1+\tau_{0} s^{\alpha}\right)\right\} \bar{e}=0
$$

The above equation can be factorized as

$$
\left(\nabla^{2}-k_{1}^{2}\right)\left(\nabla^{2}-k_{2}^{2}\right) \bar{e}=0
$$

where $k_{1}^{2}$ and $k_{2}^{2}$ are the roots with positive real parts of the characteristic equation

$$
k^{4}-k^{2}\left[s^{2}+(1+\varepsilon)\left(s+\tau_{0} s^{\alpha+1}\right)\right]+s^{3}\left(1+\tau_{0} s^{\alpha}\right)=0
$$


where $k_{1}^{2}$ and $k_{2}^{2}$ are given by

$$
\begin{aligned}
& k_{1}^{2}=\frac{s}{2}\left\{s+(1+\varepsilon)\left(1+\tau_{0} s^{\alpha}\right)+\sqrt{\left[s+(1+\varepsilon)\left(1+\tau_{0} s^{\alpha}\right)\right]^{2}-4 s\left(1+\tau_{0} s^{\alpha}\right)}\right\} \\
& k_{2}^{2}=\frac{s}{2}\left\{s+(1+\varepsilon)\left(1+\tau_{0} s^{\alpha}\right)-\sqrt{\left[s+(1+\varepsilon)\left(1+\tau_{0} s^{\alpha}\right)\right]^{2}-4 s\left(1+\tau_{0} s^{\alpha}\right)}\right\}
\end{aligned}
$$

Due to linearity, the solution to equation (3.4) has the form

$$
\bar{e}=\bar{e}_{1}+\bar{e}_{2}
$$

where $\bar{e}_{i}$ is the solution to the following equation

$$
\left(\nabla^{2}-k_{i}^{2}\right) \bar{e}_{i}=0 \quad i=1,2
$$

The above equation can be written as

$$
\frac{\partial^{2} \bar{e}_{i}}{\partial r^{2}}+\frac{2}{r} \frac{\partial \bar{e}_{i}}{\partial r}-k_{i}^{2} \bar{e}_{i}=0
$$

Taking the substitution

$$
\bar{e}_{i}=\frac{g_{i}}{\sqrt{r}}
$$

the above equation reduces to

$$
r^{2} \frac{\partial^{2} g_{i}}{\partial r^{2}}+r \frac{\partial g_{i}}{\partial r}-\left(k^{2} r^{2}+\frac{1}{4}\right) g_{i}=0
$$

This is the modified Bessel equation whose solution is

$$
g_{i}=A_{i} k_{i}^{2} I_{1 / 2}\left(k_{i} r\right)+B_{i} k_{i}^{2} K_{1 / 2}\left(k_{i} r\right)
$$

Collecting the above results, the solution to (3.7) can be written as

$$
e_{i}=\frac{1}{\sqrt{r}}\left[A_{i} k_{i}^{2} I_{1 / 2}\left(k_{i} r\right)+B_{i} k_{i}^{2} K_{1 / 2}\left(k_{i} r\right)\right]
$$

where $A_{i}$ and $B_{i}, i=1,2$ are parameters to be determined from the boundary conditions and $I_{\mu}(z), K_{\mu}(z)$ are the modified Bessel functions of the first and second kinds of the order $\mu$, respectively.

Similarly, we can show that

$$
\theta_{i}=\frac{1}{\sqrt{r}}\left[A_{i}^{*} k_{i}^{2} I_{1 / 2}\left(k_{i} r\right)+B_{i}^{*} k_{i}^{2} K_{1 / 2}\left(k_{i} r\right)\right]
$$

Substituting (3.8) and (3.9) into equation (3.3), we get

$$
A_{i}^{*}=A_{i}\left(k_{i}^{2}-s^{2}\right) \quad B_{i}^{*}=B_{i}\left(k_{i}^{2}-s^{2}\right)
$$

Substituting (3.10) into equation (3.9), one obtains

$$
\theta_{i}=\frac{1}{\sqrt{r}}\left[A_{i}\left(k_{i}^{2}-s^{2}\right) I_{1 / 2}\left(k_{i} r\right)+B_{i}\left(k_{i}^{2}-s^{2}\right) K_{1 / 2}\left(k_{i} r\right)\right]
$$


Thus we obtain

$$
\begin{aligned}
& \bar{e}=\frac{1}{\sqrt{r}} \sum_{i=1}^{2}\left[A_{i} k_{i}^{2} I_{1 / 2}\left(k_{i} r\right)+B_{i} k_{i}^{2} K_{1 / 2}\left(k_{i} r\right)\right] \\
& \bar{\theta}=\frac{1}{\sqrt{r}} \sum_{i=1}^{2}\left[A_{i}\left(k_{i}^{2}-s^{2}\right) I_{1 / 2}\left(k_{i} r\right)+B_{i}\left(k_{i}^{2}-s^{2}\right) K_{1 / 2}\left(k_{i} r\right)\right]
\end{aligned}
$$

Differentiating (3.12) with respect to $r$ and substituting into $(3.2)_{1}$, gives

$$
\bar{u}=\frac{1}{\sqrt{r}} \sum_{i=1}^{2}\left[A_{i} k_{i} I_{3 / 2}\left(k_{i} r\right)-B_{i} k_{i} K_{3 / 2}\left(k_{i} r\right)\right]
$$

Differentiating $(3.12)_{2}$ and (3.13) with respect to $r$, gives

$$
\begin{aligned}
& \frac{\partial \bar{\theta}}{\partial r}=\frac{1}{\sqrt{r}} \sum_{i=1}^{2}\left[A_{i} k_{i}\left(k_{i}^{2}-s^{2}\right) I_{3 / 2}\left(k_{i} r\right)-B_{i} k_{i}\left(k_{i}^{2}-s^{2}\right) K_{3 / 2}\left(k_{i} r\right)\right] \\
& \frac{\partial \bar{u}}{\partial r}=\frac{1}{\sqrt{r}} \sum_{i=1}^{2}\left\{A_{i} k_{i}\left[k_{i} I_{1 / 2}\left(k_{i} r\right)-\frac{2}{r} I_{3 / 2}\left(k_{i} r\right)\right]+B_{i} k_{i}\left[k_{i} K_{1 / 2}\left(k_{i} r\right)+\frac{2}{r} K_{3 / 2}\left(k_{i} r\right)\right]\right\}
\end{aligned}
$$

Substituting (3.12) and (3.14) 2 into equation $(2.3)_{3}$, gives

$$
\sigma_{r r}=\frac{1}{\sqrt{r}} \sum_{i=1}^{2}\left\{A_{i}\left[\beta^{2} s^{2} I_{1 / 2}\left(k_{i} r\right)-\frac{4}{r} k_{i} I_{3 / 2}\left(k_{i} r\right)\right]+B_{i}\left[\beta^{2} s^{2} K_{1 / 2}\left(k_{i} r\right)+\frac{4}{r} k_{i} K_{3 / 2}\left(k_{i} r\right)\right]\right\}
$$

Using equation $(3.2)_{4}$, boundary conditions (2.4) can be written in the Laplace transform as

$$
\begin{array}{ll}
\bar{u}(a, s)=0 & \frac{\partial \bar{\theta}(a, s)}{\partial r}=0 \\
\bar{\sigma}_{r r}(b, s)=0 & \bar{\theta}(b, s)=\bar{f}(s)
\end{array}
$$

Applying boundary conditions (3.16) into equations $(3.12)_{2},(3.13),(3.14)_{1}$ and (3.15), gives

$$
\begin{aligned}
& \sum_{i=1}^{2}\left[A_{i} k_{i} I_{3 / 2}\left(k_{i} a\right)-B_{i} k_{i} K_{3 / 2}\left(k_{i} a\right)\right]=0 \\
& \sum_{i=1}^{2}\left[A_{i} k_{i}\left(k_{i}^{2}-s^{2}\right) I_{3 / 2}\left(k_{i} a\right)-B_{i} k_{i}\left(k_{i}^{2}-s^{2}\right) K_{3 / 2}\left(k_{i} a\right)\right]=0 \\
& \sum_{i=1}^{2}\left\{A_{i}\left[\beta^{2} s^{2} I_{1 / 2}\left(k_{i} b\right)-\frac{4}{b} k_{i} I_{3 / 2}\left(k_{i} b\right)\right]+B_{i}\left[\beta^{2} s^{2} K_{1 / 2}\left(k_{i} b\right)+\frac{4}{b} k_{i} K_{3 / 2}\left(k_{i} b\right)\right]\right\}=0 \\
& \sum_{i=1}^{2}\left[A_{i}\left(k_{i}^{2}-s^{2}\right) I_{1 / 2}\left(k_{i} b\right)+B_{i}\left(k_{i}^{2}-s^{2}\right) K_{1 / 2}\left(k_{i} b\right)\right]=\sqrt{b} \bar{f}(s)
\end{aligned}
$$

The above equations can be put in the following form

$$
\begin{aligned}
& a_{11} A_{1}+a_{12} B_{1}+a_{13} A_{2}+a_{14} B_{2}=0 \\
& a_{21} A_{1}+a_{22} B_{1}+a_{23} A_{2}+a_{24} B_{2}=0 \\
& a_{31} A_{1}+a_{32} B_{1}+a_{33} A_{2}+a_{34} B_{2}=0 \\
& a_{41} A_{1}+a_{42} B_{1}+a_{43} A_{2}+a_{44} B_{2}=\sqrt{b} \bar{f}(s)
\end{aligned}
$$


where

$$
\begin{array}{ll}
a_{11}=k_{1} I_{3 / 2}\left(k_{1} a\right) & a_{12}=-k_{1} K_{3 / 2}\left(k_{1} a\right) \\
a_{13}=k_{2} I_{3 / 2}\left(k_{2} a\right) & a_{14}=-k_{2} K_{3 / 2}\left(k_{2} a\right) \\
a_{21}=k_{1}\left(k_{1}^{2}-s^{2}\right) I_{3 / 2}\left(k_{1} a\right) & a_{12}=-k_{1}\left(k_{1}^{2}-s^{2}\right) K_{3 / 2}\left(k_{1} a\right) \\
a_{23}=k_{2}\left(k_{2}^{2}-s^{2}\right) I_{3 / 2}\left(k_{2} a\right) & a_{24}=-k_{2}\left(k_{2}^{2}-s^{2}\right) K_{3 / 2}\left(k_{2} a\right) \\
a_{31}=\beta^{2} s^{2} I_{1 / 2}\left(k_{1} b\right)-\frac{4}{b} k_{1} I_{3 / 2}\left(k_{1} b\right) & a_{32}=\beta^{2} s^{2} K_{1 / 2}\left(k_{1} b\right)+\frac{4}{b} k_{1} K_{3 / 2}\left(k_{1} b\right) \\
a_{33}=\beta^{2} s^{2} I_{1 / 2}\left(k_{2} b\right)-\frac{4}{b} k_{2} I_{3 / 2}\left(k_{2} b\right) & a_{34}=\beta^{2} s^{2} K_{1 / 2}\left(k_{2} b\right)+\frac{4}{b} k_{2} K_{3 / 2}\left(k_{2} b\right) \\
a_{41}=\left(k_{1}^{2}-s^{2}\right) I_{1 / 2}\left(k_{1} b\right) & a_{42}=\left(k_{1}^{2}-s^{2}\right) K_{1 / 2}\left(k_{1} b\right) \\
a_{43}=\left(k_{2}^{2}-s^{2}\right) I_{1 / 2}\left(k_{2} b\right) & a_{44}=\left(k_{2}^{2}-s^{2}\right) K_{1 / 2}\left(k_{2} b\right)
\end{array}
$$

Solving the above equations, we obtain

$$
\begin{aligned}
& A_{1}=-\frac{1}{\Gamma}\left[a_{12}\left(a_{23} a_{34}-a_{24} a_{33}\right)+a_{13}\left(a_{24} a_{32}-a_{22} a_{34}\right)+a_{14}\left(a_{22} a_{33}-a_{23} a_{32}\right)\right] \sqrt{b} \bar{f}(s) \\
& B_{1}=\frac{1}{\Gamma}\left[a_{11}\left(a_{23} a_{34}-a_{24} a_{33}\right)+a_{13}\left(a_{24} a_{31}-a_{21} a_{34}\right)+a_{14}\left(a_{21} a_{33}-a_{23} a_{31}\right)\right] \sqrt{b} \bar{f}(s) \\
& A_{2}=-\frac{1}{\Gamma}\left[a_{11}\left(a_{22} a_{34}-a_{24} a_{32}\right)+a_{12}\left(a_{24} a_{31}-a_{21} a_{34}\right)+a_{14}\left(a_{21} a_{32}-a_{22} a_{31}\right)\right] \sqrt{b} \bar{f}(s) \\
& B_{2}=\frac{1}{\Gamma}\left[a_{11}\left(a_{22} a_{33}-a_{23} a_{32}\right)+a_{12}\left(a_{23} a_{31}-a_{21} a_{33}\right)+a_{13}\left(a_{21} a_{32}-a_{22} a_{31}\right)\right] \sqrt{b} \bar{f}(s)
\end{aligned}
$$

where

$$
\begin{aligned}
\Gamma= & a_{11}\left[a_{22}\left(a_{33} a_{44}-a_{34} a_{43}\right)+a_{23}\left(a_{34} a_{42}-a_{32} a_{44}\right)+a_{24}\left(a_{32} a_{43}-a_{33} a_{42}\right)\right] \\
& -a_{12}\left[a_{21}\left(a_{33} a_{44}-a_{34} a_{43}\right)+a_{23}\left(a_{34} a_{41}-a_{31} a_{44}\right)+a_{24}\left(a_{31} a_{43}-a_{33} a_{41}\right)\right] \\
& +a_{13}\left[a_{21}\left(a_{32} a_{44}-a_{34} a_{42}\right)+a_{22}\left(a_{34} a_{41}-a_{31} a_{44}\right)+a_{24}\left(a_{31} a_{42}-a_{32} a_{41}\right)\right] \\
& -a_{14}\left[a_{21}\left(a_{32} a_{43}-a_{33} a_{42}\right)+a_{22}\left(a_{33} a_{41}-a_{31} a_{43}\right)+a_{23}\left(a_{31} a_{42}-a_{32} a_{41}\right)\right]
\end{aligned}
$$

\section{Inversion of the Laplace transform}

Let $\bar{f}(s)$ be the Laplace transform of $f(t)$. The inversion formula for the Laplace transform has the form (Honig and Hirdes, 1984)

$$
f(t)=\frac{\mathrm{e}^{d t}}{2 \pi} \int_{-\infty}^{\infty} \mathrm{e}^{\mathrm{i} t y} \bar{f}(d+\mathrm{i} y) d y
$$

where $d$ is a number greater than all the real parts of the singularities of $\bar{f}(s)$.

Using Fourier series over the interval $[0,2 L]$, we get (Honig and Hirdes, 1984)

$$
f(t) \approx f_{N}(t)=\frac{1}{2} c_{0}+\sum_{k=1}^{N} c_{k} \quad \text { for } \quad 0 \leqslant t \leqslant 2 L
$$

where

$$
c_{k}=\frac{\mathrm{e}^{d t}}{L} \operatorname{Re}\left[\mathrm{e}^{\mathrm{i} k \pi t} \bar{f}\left(d+\frac{\mathrm{i} k \pi}{L}\right)\right]
$$

The 'Korrecktur' method has been used to reduce the discretization error while the $\varepsilon$-algorithm has been used to reduce the truncation error (Honig and Hirdes, 1984). 


\section{Numerical results}

Copper has been chosen for purposes of numerical evaluations. The constants of the considered problem are shown in Table 1.

Table 1

\begin{tabular}{|l|l|l|l|}
\hline$k=386 \mathrm{~W} /(\mathrm{m} \mathrm{K})$ & $\alpha_{t}=1.78 \cdot 10^{-5} \mathrm{~K}^{-1}$ & $c_{E}=381 \mathrm{~J} /(\mathrm{kg} \mathrm{K})$ & $\eta=8886.73$ \\
\hline$\mu=3.86 \cdot 10^{10} \mathrm{~kg} /\left(\mathrm{m} \mathrm{s}^{2}\right)$ & $\lambda=7.76 \cdot 10^{10} \mathrm{~kg} /\left(\mathrm{m} \mathrm{s}^{2}\right)$ & $\rho=8954 \mathrm{~kg} / \mathrm{m}^{3}$ & $T_{0}=293 \mathrm{~K}$ \\
\hline$\varepsilon=0.0168$ & $\tau_{0}=0.025 \mathrm{~s}$ & & \\
\hline
\end{tabular}

The computations have been carried out for a function $f(t)$ given by

$$
f(t)=H(t) \quad \text { for which } \quad \bar{f}(s)=\frac{1}{s}
$$

The computations have been carried out for one value of time, namely $t=0.05$, and two values of $\alpha$, namely $\alpha=0.5$ and $\alpha=1$. The temperature, displacement and stress distributions have been obtained and plotted as shown in Figs. 1, 2 and 3, respectively.

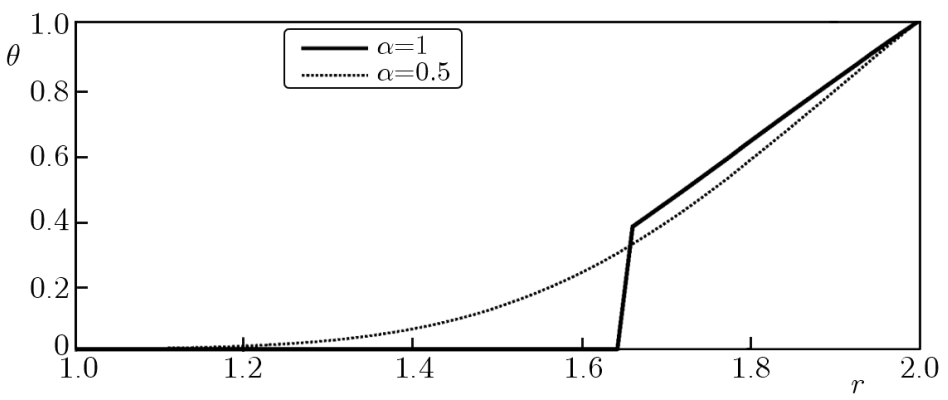

Fig. 1. Temperature distribution for $t=0.05$

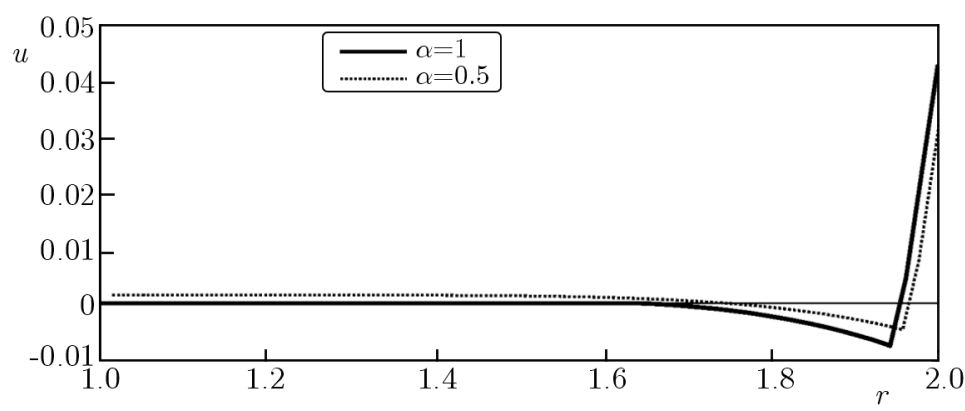

Fig. 2. Displacement distribution for $t=0.05$

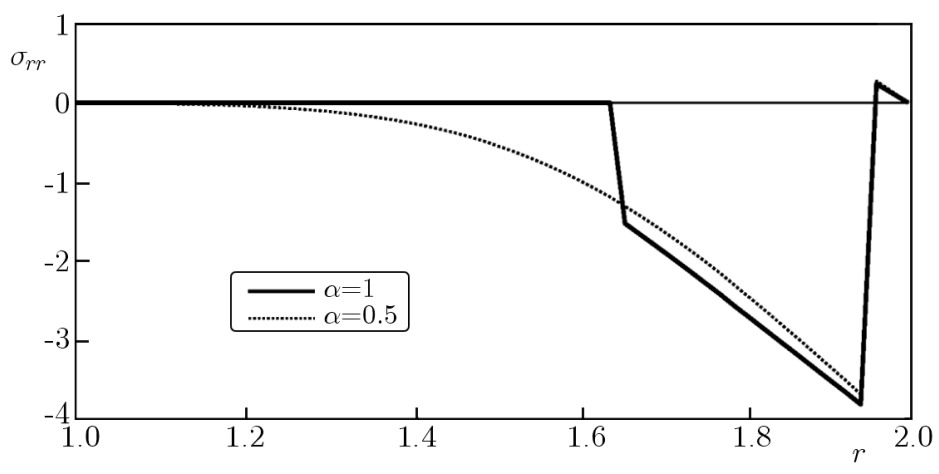

Fig. 3. Stress distribution for $t=0.05$ 
Next, the computations have been carried out for one value of $\alpha$, namely $\alpha=0.99$, and two values of time, $t=0.05$ and $t=0.1$. The temperature, displacement and stress distributions have been obtained and plotted as shown in Figs. 4, 5 and 6, respectively.

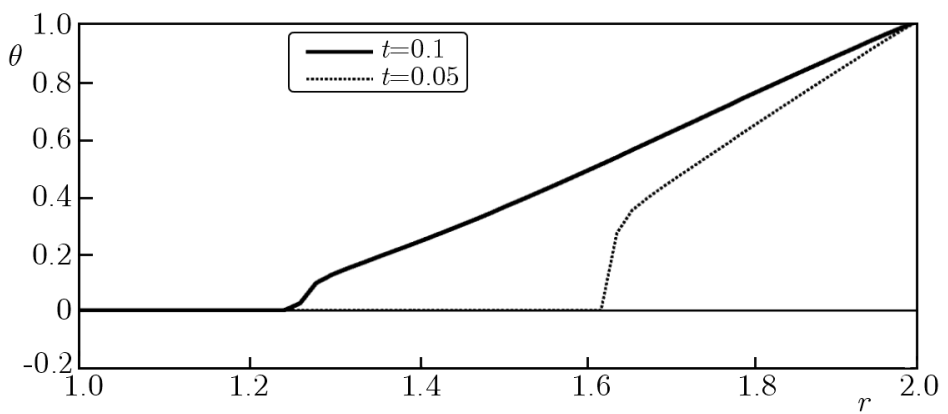

Fig. 4. Temperature distribution for $\alpha=0.99$

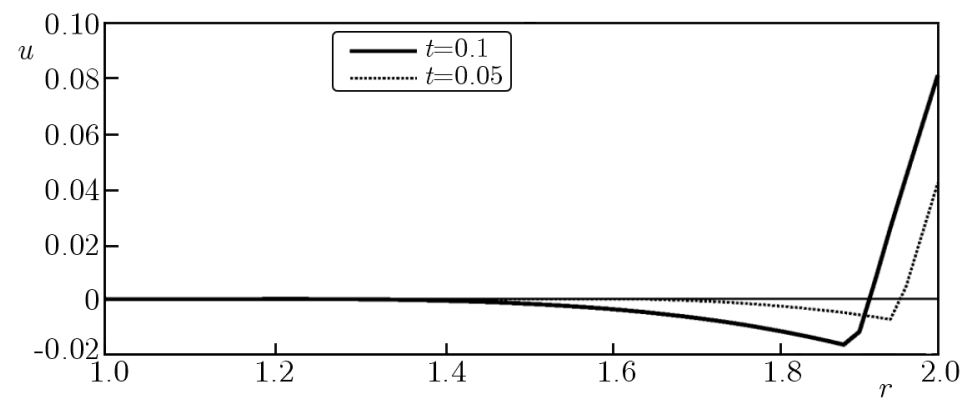

Fig. 5. Displacement distribution for $\alpha=0.99$

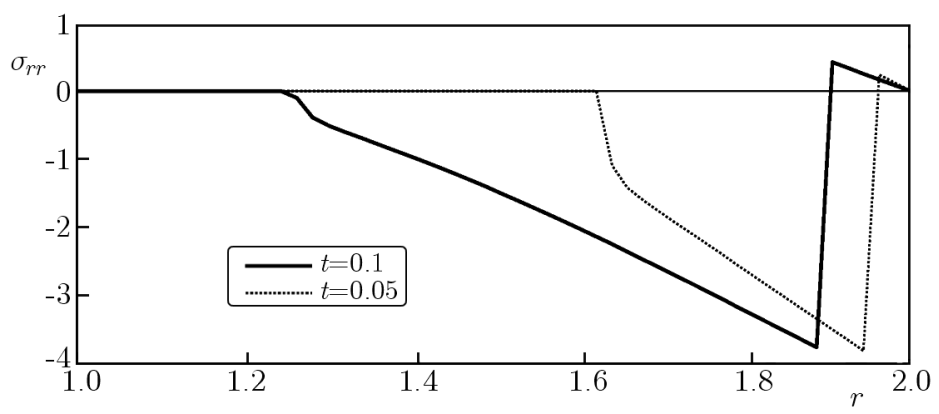

Fig. 6. Stress distribution for $\alpha=0.99$

For the pervious steps, FORTRAN programming language has been used on a personal computer. The maintained accuracy has been 5 digits for the numerical program.

\section{Conclusions}

The computations show that:

- For $\alpha=0.5$, the solution behaves like in the coupled theory of thermoelasticity where the velocity of the wave is infinite, but for $\alpha=1$ the solution becomes that of the generalized theory of thermoelasticity.

- For $\alpha \approx 1$, the solution seems to behave like in the generalized theory of thermoelasticity. This result is very important since the new theory may preserve the advantage of the generalized theory that the velocity of waves is finite. It is difficult to say whether the solution for $\alpha$ approaching 1 has a jump at the wave front or it is continuous with very fast changes (Povstenko, 2011). This aspect invites further investigation. 


\section{References}

1. Adolfsson K., Enelund M., Olsson P., 2005, On the fractional order model of viscoelasticity, Mechanics of Time Dependent Materials, 9, 15-34

2. Biот M., 1956, Thermoelasticity and irreversible thermo-dynamics, Journal of Applied Physics, 27, 240-253

3. CAputo M., 1974, Vibrations on an infinite viscoelastic layer with a dissipative memory, Journal of the Acoustical Society of America, 56, 897-904

4. Caputo M., Mainardi F., 1971a, A new dissipation model based on memory mechanism, Pure and Applied Geophysics, 91, 134-147

5. Caputo M., Mainardi F., 1971a, Linear model of dissipation in anelastic solids, Rivis ta del Nuovo Cimento, 1, 161-198

6. Hilfer R., 2000, Applications of Fractional Calculus in Physics, World Scientific Publishing, Singapore

7. Honig G., Hirdes U., 1984, A method for the numerical inversion of the Laplace transform, Journal of Computational and Applied Mathematics, 10, 113-132

8. Kaczorek T., 2011, Selected Problems of Fractional Systems Theory, Springer, Berlin

9. Kaczorek T., Rogowski K., 2015, Fractional Linear Systems and Electrical Circuits, Springer, Berlin

10. Lord H., Shulman Y., 1967, A generalized dynamical theory of thermo-elasticity, Journal of the Mechanics and Physics of Solids, 15, 299-309

11. Podlubny I., 1998, Fractional Differential Equations, Academic Press, NY

12. Povstenko Y.Z., 2005, Fractional heat conduction and associated thermal stress, Journal of Thermal Stresses, 28, 83-102

13. Povstenko Y.Z., 2009, Thermoelasticity that uses fractional heat conduction equation, Journal of Mathematical Sciences, 162, 296-305

14. Povstenko Y.Z., 2011, Fractional Cattaneo-type equations and generalized thermoelasticity, Journal of Thermal Stresses, 34, 97-114

15. Raslan W., 2015, Application of fractional order theory of thermoelasticity in a thick plate under axisymmetric temperature distribution, Journal of Thermal Stresses, 38, 733-743

16. Sherief H., AbD El-LAtief A.M., 2014a, Application of fractional order theory of thermoelasticity to a $1 \mathrm{D}$ problem for a half-space, ZAMM, 94, 509-515

17. Sherief H., ABD El-LAtief A.M., 2014b, Application of fractional order theory of thermoelasticity to a 2D problem for a half-space, Applied Mathematics and Computation, 248, 584-592

18. Sherief H., Abd El-Latief A.M., 2015, A one-dimensional fractional order thermoelastic problem for a spherical cavity, Mathematics and Mechanics of Solids, 20, 512-521

19. Sherief H., Allam M., El-Hagary M., 2011, Generalized theory of thermoviscoelasticity and a half-space problem, International Journal of Thermophysics, 32, 1271-1295

20. Sherief H., El-Maghraby N., 2003, An internal penny-shaped crack in an infinite thermoelastic solid, Journal of Thermal Stresses, 26, 333-352

21. Sherief H., El-Maghraby N., 2005, A mode I crack problem for an infinite space in generalized thermoelasticity, Journal of Thermal Stresses, 28, 465-484

22. Sherief H., El-Sayed A.M.A., Abd El-Latief A.M., 2010, Fractional order theory of thermoelasticity, International Journal of Solids and Structures, 47, 269-275

23. Sherief H., El-Sayed A.M.A., Behiry S.H., Raslan W.E., 2012, Using fractional derivatives to generalize the Hodgkin-Huxley model, Fractional Dynamics and Control, Springer, 275-282 
24. Sherief H., EzzAT M., 1994, Solution of the generalized problem of thermoelasticity in the form of series of functions, Journal of Thermal Stresses, 17, 75-95

25. Sherief H., HAmza F., 1994, Generalized thermoelastic problem of a thick plate under axisymmetric temperature distribution, Journal of Thermal Stresses, 17, 435-452

26. Sherief H., Hamza F., 1996, Generalized two-dimensional thermoelastic problems in spherical regions under axisymmetric distributions, Journal of Thermal Stresses, 19, 55-76

27. Sherief H., Hamza F., El-SAyed A., 2005, Theory of generalized micropolar thermoelasticity and an axisymmetric half-space problem, Journal of Thermal Stresses, 28, 409-437

28. Sherief H., Hussein E., 2012, A mathematical model for short-time filtration in poroelastic media with thermal relaxation and two temperatures, Transport in Porous Media, 91, 199-223

29. Tenreiro J., Alexandra M., Trujillo J., 2013, Science metrics on fractional calculus development since 1966, Fractional Calculus and Applied Analysis, 16, 479-500

Manuscript received March 22, 2014; accepted for print September 22, 2015 